\title{
On the Formation of Dissipative Spatial Patterns of Charge Carriers in Biosystems $\left.{ }^{*}\right)$.
}

\author{
A. S. ESPERIDiÃo ( $\left.{ }^{1}\right)$, Á. R. VASCONCELlos $\left({ }^{2}\right)$ and R. Luzzi ${ }^{(2)}$ \\ (') Instituto de Física, Universidade Federal da Bahia - 40210-340 Salvador, Bahia, Brasil \\ (2) Instituto de F'́rica "Gleb Wataghin", Unicamp - 13083-970 Campinas, São Paulo, Brasil
}

(ricevuto il 5 Dicembre 1994; approvato il 5 Luglio 1995)

\begin{abstract}
Summary. - It is shown that in systems like large aggregates of biological molecules, population inversion of charge carriers, for example as produced by photoexcitation processes, may have competitive advantage beyond critical levels of excitation to produce ordered spatial structures (morphological transitions). In our analysis electromagnetic radiation transfers electrons from bonding states into a continuum of itinerant antibonding states in a $p$-type doped sample. In this system, in which energy is pumped continuously by an external source, the interplay of collective and dissipative processes can be responsible for the condensation of a self-organized spatially ordered structure. The study we present here is carried out resorting to the powerful nonequilibrium statistical operator method, thus showing that it can be provide a mechano-statistical formalism at the microscopic level for the treatment of Prigogine's synergetic dissipative structures.

PACS 87.10 - General, theoretical and mathematical biophysics (including logic of biosystems, quantum biology, and relevant aspects of thermodynamics, information theory, cybernetics, and bionics).
\end{abstract}

PACS 05.70.Ln - Nonequilibrium thermodynamics, irreversible processes.

\section{1. - Introduction.}

Spatial pattern formation is a subject of increasing interest in fields like chemical-physics, hydrodynamics, and biology, e.g., the area of embryology. Recent extensive review articles are due to Meinhardt [1] and Cross and Hohenberg [2]. In these cases one is dealing with open systems in far-from-equilibrium conditions which are susceptible to develop spontaneous symmetry breaking and self-organization. This is a particular case of the so-called, after Prigogine, dissipative structures [3-5], examples of which are the different kind of flow patterns in hydrodynamics, like Bénard cells in thermal convection, and Taylor vortices between rotating cilinders [6].

$\left.{ }^{*}\right)$ The authors of this paper have agreed to not receive the proofs for correction. 
Additional examples are the formation and maintenance of self-organization in physico-chemical systems sustaining chemical or biochemical reaction chains [5-8], as well as morphological transitions that may develop as a result of diffusion instability of the kind already suggested in Turing's pioneering paper [9].

Dissipative structures in chemical networks, arising out of the different possibilities of nonlinearities, may appear in a very large number of circumstances and they are of special interest for modern biology. Thus, the analysis and understanding of dissipative structures in physical and physico-chemical systems is of importance for modelling biological systems. Such models are worth exploring as much as possible, if only to set their valid general characteristics and limitations.

The large diversity of dissipative structures that may appear in open nonequilibrium dissipative macroscopic physico-chemical and biological systems have a common phenomenological description under generalized irreversible thermodynamies [4]. This discipline brings into evidence the common features and origin of these phenomena, whose main characteristic is that they are expected to occur in a large class of open macroscopic dynamical systems governed by nonlinear laws of evolution, where structural transitions may arise in far-from-equilibrium conditions. In this nonlinear regime, the dissipative structure is stabilized beyond a critical point where the open macroscopic system is driven by external sources. The emerging self-organized structure may assume the form of [5] i) multiple steady states (e.g., lasers; semiconductors with negative differential conductivity; optically bistable semiconductors), or ii) temporal order (e.g., chemical and biological clocks, like Belousov-Zhabotinskii autocatalytic chemical reactions), and iii) spatial ordering (e.g., chemical waves; Liesegang rings in sedimentary rocks).

As already remarked Turing's model belongs to category iii). Turing conjectured that the development of structure in biology (morphogenesis) may find a physical basis in the instability of certain reacting systems with respect to symmetry-breaking perturbations. These need be open systems under the action of external sources pumping energy on them, for example the case of photoexcitation through illumination with electromagnetic radiation of different wavelength, say, visible, ultraviolet, microwaves, etc. Microwaves, in particular, are strongly absorbed in water, which represents a most important constituent of biological systems. Coupling of photoprocesses with electronic excitation and transport may allow for the possible emergence of dissipative spatial structures mantained by ambient illumination, and thus to have an important role in biomorphogenesis.

We address here a question of this kind for the case of the model described in sect. 3. For dealing with this type of problems, besides the phenomenological foundations provided by generalized irreversible thermodynamics, kinetic and stochastic theories are available $[5,8]$. Here we proposed a mechano-statistical treatment, possible for the case of many-body systems with a Hamiltonian dynamics, based on the Nonequilibrium Statistical Operator Method (NSOM). Robertson[10] and Zubarev [11] have provided elegant, and already classic, versions of the NSOM, and, more recently, we have shown that the different approaches can be put under a unifying description using Jaynes' maximum entropy formalism (Maxent) with ad hoc hypothesis [12], as briefly reviewed in next section.

The Maxent-NSOM provides mechano-statistical foundations for generalized irreversible thermodynamics [12], and even beyond, encompassing extended irreversible thermodynamics [13], removing the restriction of local equilibrium and linear flux-force relationships [14]. As is discussed in ref.[12] and [15], this statistical 
formalism appears to be encompassed in Jaynes' Predictive Statistical Mechanics (PSM) [16], which is based on Bayesian inference [17]. The thermodynamic theory derived from this Maxent-NSOM is referred to as informational statistical thermodynamics [18]. Furthermore, the Maxent-NSOM leads to the construction of a quantum nonlinear transport theory of large scope $[12,19]$. It provides the nonlinear equations of evolution of the macroscopic state of the system and, therefore, the possibility to evidence and describe dissipative structures. This is in fact the case in the analysis presented in next sections, where we use the Maxent-NSOM in Zubarev's approach adapted for the treatment of open systems [11].

In our model electrons are photoexcited from bonding molecular orbitals into itinerant antibonding states, producing a double plasma of electrons (in antibonding states) and holes (the unoccupied bonding orbitals) in the ionic background. After a transient time a nonequilibrium steady state is established corresponding to a stationary concentration, $n$, of photoinjected carriers (mobile electrons and holes) which depends on the source power. This is a homogeneous state of the plasma, which is the so-called thermodynamic branch of solutions, i.e. the one that develops continuously from the point of equilibrium along with the increasing power of the source of radiation. It is, according to Prigogine's theorem of minimum entropy production $[4,5]$ a stable solution in the strictly linear regime around equilibrium, but it may become unstable when the system is driven sufficiently far from equilibrium, leading towards a bifurcation point with the emergence of a new branch of solutions corresponding to the formation of a dissipative structure.

To test the stability of the homogeneous solution we increase the original set of nonlinear generalized transport equations, with those for an inhomogeneous spatial distribution of carriers characterized by the corresponding Fourier components of wave vector, $\boldsymbol{Q}$, with $\boldsymbol{Q}$ running over the reciprocal space. We perform linear stability analysis which shows that for the case of $p$-type doped samples (i.e. extrinsic-type materials with the presence of mobile holes) under certain conditions it may appear a point of instability (bifurcation point) of the uniform solution against the formation of a fluctuation in the carrier density with a Fourier amplitude $n(\boldsymbol{Q})$.

An electronic stationary charge density wave emerges at the critical point. Moreover, it is quite plausible that the background of positive ions (kept rigid in our model) will follow the electronic wave, thus producing a conformational transition. We are in the presence of a nonthermal transition in the sense that the energy pumped on the system by the external source is applied by the system to the production of an ordered structure instead of increasing thermal agitation. The ordered structure, as shown by the analysis described in next sections, is a result of a self-organizing outcome, which is the result of the interplay of collective effects among the photoexcited carriers together with dissipative effects produced by recombination processes, while the system is under the action of the pumping effects of external illumination.

\section{2. - Theoretical background.}

For the sake of completeness, we briefly review in this section the Nonequilibrium Statistical Operator Method (NSOM) used in the study presented in next section. The Maxent-NSOM appears to be a very powerful formalism for the theoretical study, starting at the microscopic level, of Prigogine's synergetic dissipative structures. 
This section may be skipped by the reader directly interested in the explicit results presented in next sections. There, application of the method allows to show how the presence of excited mobile charge carriers may lead to morphological order in biosystems, and to characterize the microscopic mechanisms associated to the phenomenon.

The Maxent-NSOM, among the different mechano-statistical methods devised to deal with irreversible processes, has, by far, an appealing structure and appears to provide very effective and practical ways for the treatment of nonlinear transport processes. This formalism has its sources in Boltzmann and Gibbs ideas, and particular approaches to it are based on either general arguments on projection operator techniques (for details see ref.[12] and [15]).

The first step, and a fundamental one, in NSOM is the choice of the basic set of variables deemed appropriate for the characterization of the macroscopic state of the system. This involves a contracted description in terms of, say, the dynamical quantities $P_{1}, P_{2}, \ldots, P_{n}$. The NSOM statistical operator (NSO) is a superoperator depending on these quantities to be indicated by $\varrho(t)$. The macroscopic (or mesoscopic) state is characterized by a point in Gibbs space (or space of thermodynamic states) composed at time $t$ of the set of macrovariables $Q_{j}(t)$, $j=1,2, \ldots, n$, which are the average values of the $P_{j}$, namely

$$
Q_{j}(t)=\operatorname{Tr}\left\{P_{j} \varrho(t)\right\},
$$

where Tr stands as usual for the trace operation. The choice of the basic variables depends on each case to be considered, but it is assisted by Bogoliubov's procedure of contraction of description based on a hierarchy of relaxation times [20], and, to it related, the ideas set forward by, among others, Mori[21], Zubarev[11] and Peletminskii [22]. They consist in introducing a separation of the total Hamiltonian into two parts, namely

$$
H=H_{0}+H^{\prime},
$$

where $H_{0}$ is the so-called "relevant» (or secular) part composed of the kinetic energies of the free subsystems and some of the interactions, namely those strong enough to have associated -in the spirit of Bogoliubov's principle-very short relaxation times (meaning those much smaller than the characteristic time scale of the experiment being considered), and possessing certain symmetry properties. The other therm, $H^{\prime}$, contains the interactions related to long-time relaxation mechanisms. The symmetry characteristics of the strong interactions depend on the problem under consideration: The required symmetry - to be called Zubarev-Peletminskii symmetry condition-is that

$$
\left[P_{j}, H_{0}\right]=\sum_{k=1}^{n} \alpha_{j k} P_{k},
$$

with $j=1,2, \ldots, n$, where $\left[P, H_{0}\right]=P H_{0}-H_{0} P$ is the commutator of quantities $P$ and $H_{0}$, and $\alpha_{j k}$ are, in an appropriate representation, $c$-numbers determined by $H_{0}$. It ought to be noticed that quantities $P_{j}$ can be dependent on the space variable, i.e. they may be local densities, and then the coefficients $\alpha$ can also be differential operators or depend on the space variable. Moreover, eq. (3) also admits coefficients $\alpha$ which are null, corresponding to the case of quantities $P$ which are conserved under 
the dynamics generated by $H_{0}$; accordingly they are acceptable basic variables, and so is $H_{0}$ itself, which falls under this condition.

Equation (3) provides a closure condition for the choice of the basic set of variables in a step-by-step procedure: First, the secular part $H_{0}$ in eq. (1) is adequately chosen in each particular problem under consideration. Second, one introduces a few dynamical variables $P$ deemed relevant for the case and next the commutators of them with $H_{0}$ are calculated. The dynamical variables-different from those already introduced-which appear in the linear combination on the right of eq. (3) are incorporated to the basic set. The procedure is repeated sequentially until a closure is attained. In ref.[14] we relate this procedure to the question of the choice of the basic macrovariables in phenomenological irreversible thermodynamies, and an example in semiconductor physics is discussed. Since the closure may not follow in a finite number of steps (e.g., see second of ref.[14]), practical application of the formalism requires the introduction of an appropriate truncation along the chain in the procedure just described, hence introducing approximations that need be evaluated in each case.

Once the basic set $\left\{P_{j}\right\}$ has been chosen, the NSO is built within the context of PSM resorting to the Maxent formalism, with memory and ad hoc hypotheses to ensure irreversibility from the outset [12,15]. In short, Gibbs' statistical entropy

$$
S_{\mathrm{G}}(t)=-\operatorname{Tr}\{\varrho(t) \ln \varrho(t)\},
$$

with $\varrho(t)$ defined in the interval $\left(t_{0}, t\right)$, where $t_{0}$ is the initial time of preparation of the system and $t$ the time when a measurement is performed, is maximized subjected to the constraints that is normalized at all times, i.e.

$$
\operatorname{Tr}\left\{\varrho\left(t^{\prime}\right)\right\}=1
$$

and that

$$
Q_{j}\left(t^{\prime}\right)=\operatorname{Tr}\left\{P_{j} \varrho\left(t^{\prime}\right)\right\} \quad(j=1,2, \ldots, n),
$$

for $t_{0} \leqslant t^{\prime} \leqslant t$. Resorting to the standard procedure of using Lagrange method, but introducing particular ad hoc hypotheses on the form of the Lagrange multipliers, we obtain that $[12,15]$

$$
\varrho_{w}(t)=\exp \left[\int_{t_{0}}^{t} \mathrm{~d} t^{\prime} w\left(t, t^{\prime}\right) \ln \bar{\varrho}\left(t^{\prime}, t^{\prime}-t\right)\right],
$$

where it has been introduced the auxiliary coarse-grained NSO $\bar{\varrho}$, which plays an important role in the formalism, given by

$$
\bar{\varrho}\left(t_{1}, t_{2}\right)=\exp \left[-\phi\left(t_{1}\right)-\sum_{j=1}^{n} F_{j}\left(t_{1}\right) P_{j}\left(t_{2}\right)\right] .
$$

In this equation (8), $t_{1}$ indicates the time dependence of the quantities (arising out of the Lagrange multipliers) $\phi$ and $F_{j}$, and $t_{2}$ indicates the dependence on time of the quantities $P_{j}$ whose evolution is governed by Heisenberg equations of motion. In eq. (7) $w\left(t, t^{\prime}\right)$ is a weight function with well-defined properties $[12,15]$ which allows 1) to introduce the set of variables $F_{j}(t)$ that have the role of intensive variables thermodynamically conjugated to variables $Q_{j}(t)$ in order to generate a complete 
connection with irreversible thermodynamics, and 2) to fix an initial condition from which proceeds the irreversible evolution of the macrostate of the system. A further condition is imposed, namely the coarse-graining condition that

$$
Q_{j}(t)=\operatorname{Tr}\left\{P_{j} \varrho_{w}(t)\right\}=\operatorname{Tr}\left\{P_{j} \bar{\varrho}(t, 0)\right\},
$$

for any one of the variables of the basic set, which ensures the normalization of $\bar{\varrho}$ once $\varrho_{w}$ is normalized, i.e.

$$
\phi(t)=\ln \operatorname{Tr}\left\{\exp \left[-\sum_{j=1}^{n} F_{j}(t) P_{j}\right]\right\}
$$

and completes the objectives of items 1) and 2) above.

Furthermore, it can be shown that the NSO can be split into two parts, namely

$$
\varrho_{w}(t)=\bar{\varrho}(t, 0)+\varrho_{w}^{\prime}(t),
$$

that is, the NSO is composed of an instantaneous ( ffrozen" or coarse-grained) part $\bar{\varrho}(t, 0)$, which also defines the instantaneous average values of the basic variables as shown by eq. (9), plus another contribution $\varrho_{w}^{\prime}$ that is shown to account for the microscopic processes that produce dissipative effects in the system. The Maxent-NSOM statistical operator thus obtained satisfies a Liouville equation with a so-called Boltzmann-Prigogine symmetry, namely, the presence of an infinitesimal source that breaks its otherwise time-reversal symmetry. This is done by the presence of the weight function $w$, which ensures the irreversible evolution of the macroscopic state of the system from an initial condition, that is $\varrho_{w}\left(t_{0}\right)=$ $=\bar{\varrho}\left(t_{0}, t\right)[12,15]$.

The connection of the Maxent-NSOM with phenomenological irreversible thermodynamics is discussed elsewhere [14,18]. Finally, to close the formalism and to perform calculations of thermodynamic functions [14] and response functions [12,23], it is necessary to obtain and solve the equations of evolution for the basic macrovariables $Q_{j}(t)$. This is done by proceeding to the differentiation in time of eqs. (9) to obtain

$$
\frac{\partial}{\partial t} Q_{j}(t)=J_{j}^{(0)}(t)+J_{j}^{(1)}(t)+\mathscr{I}_{j}(t)
$$

where

$$
\begin{aligned}
& J_{j}^{(0)}(t)=\operatorname{Tr}\left\{\frac{1}{i \hbar}\left[P_{j} H_{0}\right] \bar{\varrho}(t)\right\}, \\
& J_{j}^{(1)}(t)=\operatorname{Tr}\left\{\frac{1}{i \hbar}\left[P_{j} H^{\prime}\right] \bar{\varrho}(t)\right\}, \\
& \mathscr{T}_{j}(t)=\operatorname{Tr}\left\{\frac{1}{i \hbar}\left[P_{j}, H^{\prime}\right] \bar{\varrho}_{w}(t)\right\} .
\end{aligned}
$$

The quantity of eq. (13c) is a collision operator which is of fundamental relevance to the characterization of the dissipative processes that develop in the media. It is 
extremely difficult to handle, but it can be shown that it can be expressed in terms of an infinite series of partial-collision operators, which are instantaneous in time and organized in increasing order $n$ in the strengths of the interactions contained in $H^{\prime}$ [19]. The lowest order of approximation consists in retaining only the term with $n=2$. This is a local-in-time (memoryless) approximation, sometimes referred to as the quasi-linear theory of relaxation [24], but which we have called second-order approximation in relaxation theory, SOART for short[19]. In SOART the collision operator $\mathscr{I}_{j}$ is approximated by the second-order partial-collision operator $J_{j}^{(2)}$ given by

$$
\begin{aligned}
J_{j}^{(2)}(t)=\left(\frac{1}{i \hbar}\right)^{2} \int_{0}^{t} \mathrm{~d} t^{\prime} \mathscr{W}\left(t, t^{\prime}\right) & \operatorname{Tr}\left\{\left[H^{\prime}\left(t^{\prime}\right),\left[H^{\prime}, P_{j}\right]\right] \bar{\varrho}(t)\right\}+ \\
+ & \frac{1}{i \hbar} \int_{t_{0}}^{t} \mathrm{~d} t^{\prime} \mathscr{W}\left(t, t^{\prime}\right) \sum_{k} \frac{\delta J_{j}^{(1)}(t)}{\delta Q_{k}(t)} \operatorname{Tr}\left\{\left[P_{k}, H^{\prime}\right] \bar{\varrho}(t)\right\},
\end{aligned}
$$

where we have defined $w\left(t, t^{\prime}\right)=\mathrm{d} \mathscr{W}\left(t, t^{\prime}\right) / \mathrm{d} t^{\prime}$ and $\delta$ indicates functional derivative.

This generalized nonlinear quantum transport theory is at the core of the treatment to deal with self-organizating dissipative structures, as is the case in following section.

\section{3. - The photoactivated morphological transition.}

Let us consider a large array of atoms forming a long chain of macromolecules in a biopolymer. According to quantum mechanics electrons bound to atoms can be found only at certain energy levels, which in large aggregates of atoms group themselves into allowed energy bands. Our model will consist of electrons in localized bonding states in a fully or partially ( $p$-type material) occupied narrow band, which we describe approximately by a parabolic band of free electrons with a very large effective mass. (The equivalent of the valence band in crystaline solids.) The itinerant higher-energy antibonding states in a nonoccupied wide band are described by a parabolic band of free electrons with a small effective mass. (The equivalent of the conduction band in crystalline solids.) The system is subjected to illumination by radiation with a large spectrum of frequencies. As a result, in photon absorption processes electrons are transferred from the bonding to the antibonding band states, thus creating electron-hole pairs, that is, an electron in the upper band and a hole, i.e. the remaining unoccupied state, in the lower band. The photoinjected excess energy received by these carriers (electrons and holes) is dissipated in recombination processes and relaxation to the vibrational modes of the system.

Under continuous illumination a steady state sets in (the transient time will depend on the intensity of the radiation). In this state there is a concentration $n$ of photoinjected pairs (each pair consisting of an electron and a hole produced by the absorption of a single photon). The system is taken to be in thermal contact with a reservoir at temperature $T_{0}$ which is kept constant by an effective homeostatic process, and we assume that the whole carrier system is in near thermal equilibrium with it, as is suggested by results obtained in the study of photoexcited semiconductors [25]. Hence, in this way the electron system is brought to a stationary and homogeneous state. This is the thermodynamic branch of solutions which, 
according to Prigogine's minimum-entropy-production theorem $[4,5]$, is a stable one in the linear regime around equilibrium. We consider next the situation when the carrier system is driven far from equilibrium, and we perform linear stability analysis [5] encompassing inhomogeneous situations. This is done considering the case of a space-dependent fluctuation in the carrier density. As expressed in the Introduction we resort to the use of the Maxent-NSOM, whose general formulation has been briefly described in last section, and Zubarev's approach to the Maxent-NSOM [11] is chosen when performing calculations. As noted, the use of the Maxent-NSOM requires to specify the system Hamiltonian and to choose a basic set of variables deemed appropriate for the description of the macroscopic state of the system. We take as basic macrovariables the carrier concentration, the temperature $T_{0}$ (which is simply a constant parameter since it was assumed thermalization with the reservoir) and, to introduce spatial inhomogeneity in the problem, we include the nondiagonal elements of the single-particle distribution matrix, namely

$$
\begin{gathered}
n_{k \boldsymbol{Q}}^{\mathrm{e}}(t)=\operatorname{Tr}\left\{C_{\boldsymbol{k}+\boldsymbol{Q}}^{\dagger} C_{\boldsymbol{k}} \varrho_{\varepsilon}(t)\right\}, \\
n_{\boldsymbol{k} \boldsymbol{Q}}^{\mathrm{h}}(t)=\operatorname{Tr}\left\{h_{-\boldsymbol{k}-\boldsymbol{Q}} h_{-\boldsymbol{k}}^{\dagger} \varrho_{\varepsilon}(t)\right\},
\end{gathered}
$$

with $C^{\dagger}(C)$ and $h^{\dagger}(h)$ representing creation (annhilation) operators of electrons and of holes in quantum-mechanical states $|\boldsymbol{k}\rangle$, respectively; $\varrho_{\varepsilon}(t)$ is Zubarev's NSO for this case; indices e and $h$ stand for electrons and holes, respectively; and $\boldsymbol{k}$ and $\boldsymbol{Q}$ run over the whole reciprocal space. The $\boldsymbol{Q}$-wave-vector Fourier amplitude of the charge density is given, in units of the electronic charge, by

$$
n(\boldsymbol{Q}, t)=\sum_{\boldsymbol{k}}\left[n_{\boldsymbol{k} \boldsymbol{Q}}^{\mathrm{e}}(t)+n_{\mathbf{k} \boldsymbol{Q}}^{\mathrm{h}}(t)\right] .
$$

Zubarev's NSO $\varrho_{\varepsilon}(t)$ is given by the expression of eq. (7) with the corresponding choice of the weight function, namely, $w\left(t, t^{\prime}\right)=\varepsilon \exp \left[\varepsilon\left(t-t^{\prime}\right)\right]$, that is to say

$$
\varrho_{\varepsilon}(t)=\exp \left[\varepsilon \int_{-\infty}^{0} \mathrm{~d} \tau \exp [\varepsilon \tau] \ln \bar{\varrho}(t+\tau, \tau)\right],
$$

where the change $\tau=t^{\prime}-t$ has been introduced, and $\varepsilon$ is a positive infinitesimal (which ensures irreversible evolution from the initial macrostate of the system) which goes to zero after the calculation of averages. The auxiliary coarse-grained statistical operator $\bar{\varrho}$ is in this case given by

$$
\begin{aligned}
\bar{\varrho}\left(t_{1}, t_{2}\right)=\exp [- & \phi\left(t_{1}\right)-\beta H_{0}\left(t_{2}\right)+\beta \mu_{\mathrm{e}}\left(t_{1}\right) N_{\mathrm{e}}\left(t_{2}\right)+\beta \mu_{n}\left(t_{1}\right) N_{n}\left(t_{2}\right)- \\
& \left.\quad-\sum_{\boldsymbol{k} \boldsymbol{Q}} F_{k \boldsymbol{Q}}^{(\mathrm{e})}\left(t_{1}\right) C_{\boldsymbol{k}+\boldsymbol{Q}}^{\dagger}\left(t_{2}\right) C_{\boldsymbol{k}}\left(t_{2}\right)-\sum_{\boldsymbol{k} \boldsymbol{Q}} F_{\boldsymbol{k} \boldsymbol{Q}}^{(\mathrm{h})}\left(t_{1}\right) h_{-\boldsymbol{k}-\boldsymbol{Q}}\left(t_{2}\right) h_{-\boldsymbol{k}}^{\dagger}\left(t_{2}\right)\right],
\end{aligned}
$$

where $\phi$ ensures the normalization of $\bar{\varrho}$ (cf. eq. (10)), $\beta=1 / k_{\mathrm{B}} T_{0}$ is the reciprocal of the temperature of the system in equilibrium with the reservoir, $k_{\mathrm{B}}$ being Boltzmann constant, $\mu_{\mathrm{e}}$ and $\mu_{\mathrm{h}}$ are the quasi-chemical potentials of electrons and of holes, respectively, and $F^{(\mathrm{e})(\mathrm{h})}$ are the Lagrange multipliers the method introduces which are those associated to the dynamical quantities whose average values are given by 
eqs. (15). $N_{\mathrm{e}}$ and $N_{\mathrm{h}}$ are the number operators for the carriers, and $H_{0}$ is the Hamiltonian of the carriers given by eq. (20a) below.

The total Hamiltonian is

$$
H=H_{0}+H^{\prime},
$$

where

$$
\begin{aligned}
& H_{0}=\sum_{k}\left(E_{\mathrm{G}}+\epsilon_{k}^{\mathrm{e}}\right) C_{k}^{\dagger} C_{k}+\sum_{k} \epsilon_{k}^{\mathrm{h}} h_{-k}^{\dagger} h_{-\boldsymbol{k}}+V(\text { Coulomb }), \\
& H^{\prime}=\sum_{k \boldsymbol{q} \gamma} G_{\gamma}(\boldsymbol{k}, \boldsymbol{q}) f_{\boldsymbol{q} \gamma} C_{k+q}^{\dagger} h_{-k}^{\dagger}+\text { h. c. }
\end{aligned}
$$

In eq. (20a) $E_{G}$ is the energy gap, i.e. the separation between the upper energy level in the bonding states (taken as the origin of the axis of energy) and the lower energy level in the antibonding states. Further, as previously indicated, the energy dispersion in both bands is taken in a parabolic band approximation, namely

$$
\epsilon_{k}^{\mathrm{e}(\mathrm{h})}=\hbar^{2} k^{2} / 2 m_{\mathrm{e}(\mathrm{h})} .
$$

$V\left(\right.$ Coulomb) is Coulomb interaction between carriers, and in $H^{\prime} f\left(f^{\dagger}\right)$ are annihilation (creation) operators of photons in states of wave vector $q$ and polarization $\gamma$, and $G$ is

$$
G_{\gamma}(\boldsymbol{k}, \boldsymbol{q})=\left(1 / m_{0} c\right) A\left(\omega_{q}\right)\left\langle\boldsymbol{k}+\boldsymbol{q}, e\left|\exp [i \boldsymbol{q} \cdot \boldsymbol{r}] \check{e}_{\gamma} \cdot \boldsymbol{p}\right| \check{e}_{\gamma} \cdot \boldsymbol{p} \mid \boldsymbol{k}, h\right\rangle,
$$

viz. the matrix element between electron and hole states of the carrier-photon interaction. In eq. (21b) $m_{0}$ is the rest electron mass, $c$ the velocity of light, $A$ the amplitude of the vector potential of the radiation field at the photon frequency $\omega_{q}, p$ the linear momentum operator of the electrons, and $\breve{e}$ a unit polarization vector. The vector potential $\boldsymbol{A}$ encompasses both fields, i.e. the one associated to the external light source and the one associated to the recombination (luminescent) field.

The system Hamiltonian of eq. (19) is composed of $H_{0}$, namely, the energy operators for the carriers, which will be treated in Landau's quasi-particle approach with Coulomb interaction dealt with in the random phase approximation (RPA) [26], and of $H^{\prime}$ that contains the interactions of the carriers with the external radiation and recombination fields of photons. The interaction of the carriers with the vibrational motion is neglected since it is only relevant to the exchanges of energy that have already led the electron system quasi-temperature to near-coincide with that of the reservoir [25] and, thus, the vibrational degrees of freedom do not play any relevant part in the study that follows.

In continuation we obtain the equations of evolution of the basic set of macrovariables resorting to the nonlinear quantum transport theory that is derived from the Maxent-NSOM[11,12] briefly described in sect. 2, and in the SOART approximation, as given by eqs. (12) together with (13a), (13b) and (14). SOART, we recall, consists in approximating the transport equations in an instantaneous (memoryless) approach, where we retain the contributions to the scattering operators only up to second order in the interactions $[11,12,24]$, i.e. as noted it consists in taking the three contributions given by eqs. (13a), (13b) and (14).

Applying the method to derive the equation of evolution for the concentration $n$, we find in stationary conditions the equation that connects the concentration $n$ with 
the intensity of the radiation source, namely

$$
\begin{aligned}
\int_{E_{\mathrm{G}} / \hbar}^{\infty} \mathrm{d} \omega_{r} \alpha\left(\omega_{r}\right)\left[1-f_{r}^{\mathrm{e}}-f_{r}^{\mathrm{h}}\right] I\left(\omega_{r}\right) / \hbar & = \\
& =\frac{2 \pi}{\hbar} \sum_{\boldsymbol{k} \boldsymbol{q} \gamma}\left|G_{\gamma}^{R}(\boldsymbol{k}, \boldsymbol{q})\right|^{2} f_{\boldsymbol{k}}^{\mathrm{e}} f_{\boldsymbol{k}}^{\mathrm{h}} \delta\left(\epsilon_{\boldsymbol{k}}^{x}+E_{\mathrm{G}}-\hbar \omega_{q \gamma}\right),
\end{aligned}
$$

where $G_{\gamma}^{R}$ is the matrix element of the recombination process (see eq. $(21 b)$ ), $\epsilon_{k}^{x}=$ $=\hbar^{2} k^{2} / 2 m_{x}$, with $m_{x}^{-1}=m_{\mathrm{e}}^{-1}+m_{\mathrm{h}}^{-1}$ being the so-called excitonic mass, $\omega_{q}$ is the frequency of the photons produced in recombination processes given by $c q / \varepsilon_{\infty}^{1 / 2}\left(\varepsilon_{\infty}\right.$ is the high-frequency dielectric constant of the system);

$$
f_{k}^{\mathrm{e}(\mathrm{h})}=\left[1+\exp \left[\beta\left(\epsilon_{k}^{\mathrm{e}(\mathrm{h})}-\mu_{\mathrm{e}(\mathrm{h})}\right)\right]\right]^{-1}
$$

is the Fermi-Dirac distribution functions of electrons (holes); $f_{r}^{\mathrm{e}(\mathrm{h})}$ are of the form of eq. (23) but taken with energies $\left(m_{\mathrm{e}(\mathrm{h})} / m_{x}\right)\left(\hbar \omega_{r}-E_{\mathrm{G}}\right)$ instead of $\epsilon_{k}^{\mathrm{e}(h)} ; \alpha\left(\omega_{r}\right)$ is the one-photon absorption coefficient at frequency $\omega_{r} ;$ and $I\left(\omega_{r}\right)$ is the spectral distribution of the intensity of the external source of radiation. Equation (22) simply reflects the balance condition in the stationary state between the number of carriers produced by illumination, the left-hand side, and the number that disappears in luminescent recombination, the right-hand side. Further, the quasi-chemical potentials $\mu_{\mathrm{e}(\mathrm{h})}$ are related to the reciprocal temperature $\beta$ and the concentrations $n_{\mathrm{e}}$ of electrons and $n_{\mathrm{h}}$ of holes, by the expressions [27]

$$
\begin{gathered}
n_{\mathrm{e}}=n_{\mathrm{e}}^{0} F_{1 / 2}\left[\beta\left(\mu_{\mathrm{e}}-E_{\mathrm{G}}\right)\right], \\
n_{\mathrm{h}}=n_{\mathrm{h}}^{0} F_{1 / 2}\left(\beta \mu_{\mathrm{h}}\right),
\end{gathered}
$$

where

$$
n_{\mathrm{e}(\mathrm{h})}^{0}=2\left[2 \pi m_{\mathrm{e}(\mathrm{h})} / \hbar^{2} \beta\right]^{3 / 2}
$$

and $F_{1 / 2}$ are Fermi functions of index one-half.

The equations of motion for the quantities of eqs. (15), calculated using eqs. (12) in SOART, after laborious calculations whose details we omit for the sake of brevity, and resorting, as noted, to the RPA, are given by

$$
\begin{aligned}
& i \hbar \frac{\partial}{\partial t} n_{k Q}^{\mathrm{e}}(t)=\Delta E_{k \boldsymbol{Q}}^{\mathrm{e}} n_{\boldsymbol{k} \boldsymbol{Q}}^{\mathrm{e}}-2 V(Q) \Delta f_{k \boldsymbol{Q}}^{\mathrm{e}} n(\boldsymbol{Q})+i B_{\boldsymbol{k} Q}^{\mathrm{e}} n_{\boldsymbol{k} Q}^{\mathrm{h}}-i B_{k \boldsymbol{Q}}^{\mathrm{h}} n_{k \boldsymbol{Q}}^{\mathrm{e}}+\mathcal{N}_{\boldsymbol{k} Q}^{\mathrm{e}}, \\
& i \hbar \frac{\partial}{\partial t} n_{k Q}^{\mathrm{h}}(t)=-\Delta E_{k Q}^{\mathrm{h}} n_{k Q}^{\mathrm{h}}+2 V(Q) \Delta f_{k Q}^{\mathrm{h}} n(\boldsymbol{Q})+i B_{k Q}^{\mathrm{h}} n_{k Q}^{\mathrm{e}}-i B_{k Q}^{\mathrm{e}} n_{k Q}^{\mathrm{h}}+\mathcal{N}_{k Q}^{\mathrm{h}},
\end{aligned}
$$

where

$$
\begin{gathered}
\Delta E_{k Q}^{\mathrm{e}(\mathrm{h})}=\epsilon_{k+Q}^{\mathrm{e}(\mathrm{h})}-\epsilon_{k}^{\mathrm{e}(\mathrm{h})}, \\
\Delta f_{k Q}^{\mathrm{e}(\mathrm{h})}=f_{k+Q}^{\mathrm{e}(\mathrm{h})}-f_{k}^{\mathrm{e}(\mathrm{h})}, \\
V(Q)=4 \pi e^{2} / \mathscr{D}_{\varepsilon_{0}} Q^{2},
\end{gathered}
$$


with $\varepsilon_{0}$ being the static dielectric constant of the medium and $\mathscr{V}$ the volume of the system. In eq. (26c), $V(Q)$ is the Fourier $Q$-component of Coulomb potential interaction between carriers.

The first two terms of eqs. (25) have their origin in collision operators $J^{(0)}$ and $J^{(1)}$ of eqs. (13a) and (13b) and are related to the changes in kinetic and potential (Coulomb) energies associated to the creation of this particular inhomogeneity. The third and fourth terms have their origin in the collision operator $J^{(2)}$ of eq. (14) and are related to the absorption of photons and recombination processes, with coefficients $B$ given by

$$
B_{k \boldsymbol{Q}}^{\mathrm{e}(\mathrm{h})}=A_{\mathrm{L}} \mathscr{G}\left(\omega_{\mathrm{L}}\right)+A_{\mathrm{R}}\left(\epsilon_{k}^{x}+E_{\mathrm{G}}\right) f_{k}^{\mathrm{e}(\mathrm{h})}+\text { same term with } \boldsymbol{k} \leftrightarrow \boldsymbol{k}+\boldsymbol{Q},
$$

where

$$
\begin{aligned}
& A_{\mathrm{L}}=\left(2 \pi^{2} \hbar e^{2} E_{\mathrm{G}} / \varepsilon_{\infty}\left(\epsilon_{k}^{x}+E_{\mathrm{G}}\right)^{2} c m_{x}\right) I_{0}, \\
& A_{\mathrm{R}}=e^{2} E_{\mathrm{G}} / \varepsilon_{\infty} \hbar c^{3} m_{x} .
\end{aligned}
$$

We have introduced a source of radiation characterized by a spectrum $I\left(\omega_{r}\right)$, that is the intensity at frequency $\omega_{r}$ for which we wrote $I\left(\omega_{r}\right)=I_{0} \mathscr{E}\left(\omega_{r}\right)$, where $\mathscr{I}$ is the (normalized) spectral distribution function of the radiation intensity having total intensity $I_{0}$. Moreover, in eq. (27), $\hbar \omega_{\mathrm{L}}=E_{\mathrm{G}}+\epsilon_{k}^{x}$ as a result of energy conservation in the process of pair production by absorption of one photon with frequency $\omega_{\mathrm{L}}$.

Finally, the terms $\mathcal{N}_{k Q}^{\mathrm{e}(\mathrm{h})}$ contain nonlinear contributions in the quantities of eqs. (15), treated in RPA, and associated with Coulomb interaction, and light absorption and recombination effects. We do not explicitly write down their lengthy expressions since they are not going to be explicitly used in the following linear analysis. (They are of relevance a posteriori for the determination of the dissipative structure whose emergence is evidenced by the linear analysis.) It suffices to say that these nonlinear terms are null for $n_{k Q}^{e(h)}=0$, i.e. in the homogeneous state.

A steady-state solution (the so-called fixed point or singular point) of eqs. (25) corresponds to $n_{k q}^{\text {e(h) }}=0$, i.e. the homogeneous state, which, as noted, is stable in the linear regime around equilibrium (weak external fields). To look for its instability in far-from-equilibrium conditions we analyse the eigenvalue spectrum of the set of linearized equations $(25)[5,28]$, that is taking in them $\mathcal{N}_{k Q}^{\mathrm{e}(\mathrm{h})}=0$ and for the carrier distributions $f_{k}^{\text {e(h) }}$ their values in the homogeneous state. In particular we consider the case of null eigenvalue corresponding to the onset of an instability against a static inhomogeneity.

We solve the linearized equations (25) for the case of null eigenvalue to obtain

$$
n_{k Q}^{\text {e(h) }}=2 V(Q) N_{k Q}^{e(h)} D_{k Q}^{-1} n(Q),
$$

where

$$
\begin{gathered}
N_{k Q}^{\mathrm{e}(\mathrm{h})}=-\Delta f_{k Q}^{\mathrm{e}(\mathrm{h})} \Delta E_{k Q}^{\mathrm{h}(\mathrm{e})}+i\left[\Delta f_{k Q}^{\mathrm{h}}-\Delta f_{k Q}^{\mathrm{e}}\right] B_{k Q}^{\mathrm{e}(\mathrm{h})}, \\
D_{k Q}=-\left(\Delta E_{k Q}^{\mathrm{e}}-i B_{k Q}^{\mathrm{h}}\right)\left(\Delta E_{k Q}^{\mathrm{h}}+i B_{k Q}^{\mathrm{e}}\right)+B_{k Q}^{\mathrm{e}} B_{k Q}^{\mathrm{h}} .
\end{gathered}
$$

Adding up both expressions in eqs. (29), the e- and h-contributions, and summing 
over $k$ we find that

$$
n(\boldsymbol{Q})\left[1-2 V(Q) \sum_{k}\left(N_{k \boldsymbol{Q}}^{\mathrm{e}}+N_{k \boldsymbol{Q}}^{\mathrm{h}}\right) D_{k \boldsymbol{Q}}^{-1}\right]=0,
$$

which besides $n(\boldsymbol{Q})=0$, the thermodynamic branch of solutions (homogeneous state), admits a nonvanishing value for $n(\boldsymbol{Q})$ (inhomogeneous solution) if the expression within the square bracket is null. It is worth noticing that the expression in the square bracket of eq. (31) is the wave-vector-dependent static dielectric function of the nonequilibrium carrier system, and, accordingly, we indicate it by $\varepsilon(\boldsymbol{Q})$. Since it is a complex quantity to put it equal to zero requires that both the real and the imaginary part be null. The imaginary part vanishes identically as it should, because it is the zero frequency value of the imaginary part of the dynamic dielectric function which is an odd function of the frequency [29]. The zero of the real part of $\varepsilon$, in the limit of small $Q$, meaning $Q^{2}$ smaller than the average value of the squared carrier quasi-momentum $\left\langle k^{2}\right\rangle$, is given by

$$
\operatorname{Re} \varepsilon(\boldsymbol{Q})=1-2 V(Q) \sum_{k}[a(k, \boldsymbol{Q})+b(k, \boldsymbol{Q})] c^{-1}(\boldsymbol{k}, \boldsymbol{Q})=0,
$$

where

$$
\begin{gathered}
a(\boldsymbol{k}, \boldsymbol{Q})=\left(\hbar^{2} \boldsymbol{k} \cdot \boldsymbol{Q} / m_{\mathrm{e}}\right)^{2}\left(\hbar^{2} \boldsymbol{k} \cdot \boldsymbol{Q} / m_{\mathrm{h}}\right)^{2}\left(f_{\boldsymbol{k}}^{\prime \mathrm{e}}+f_{k}^{\prime \mathrm{h}}\right), \\
b(\boldsymbol{k}, \boldsymbol{Q})=\left(\hbar^{2} \boldsymbol{k} \cdot \boldsymbol{Q}\right)^{2}\left(\frac{f_{\boldsymbol{k}}^{\mathrm{h}}}{m_{\mathrm{h}}}-\frac{f_{k}^{\prime \mathrm{e}}}{m_{\mathrm{e}}}\right)\left(\frac{B_{\boldsymbol{k} \boldsymbol{Q}}^{\mathrm{h}}}{m_{\mathrm{h}}}-\frac{B_{\boldsymbol{k} \boldsymbol{Q}}^{\mathrm{e}}}{m_{\mathrm{e}}}\right)\left(B_{\boldsymbol{k} \boldsymbol{Q}}^{\mathrm{e}}+B_{\boldsymbol{k} \boldsymbol{Q}}^{\mathrm{h}}\right), \\
c(\boldsymbol{k}, \boldsymbol{Q})=\left[\left(\hbar^{2} \boldsymbol{k} \cdot \boldsymbol{Q} / m_{\mathrm{e}}\right)\left(\hbar^{2} \boldsymbol{k} \cdot \boldsymbol{Q} / m_{\mathrm{e}}\right)\right]^{2}+\left[\left(\hbar^{2} \boldsymbol{k} \cdot \boldsymbol{Q}\right)\left(\frac{B_{\boldsymbol{k} \boldsymbol{Q}}^{\mathrm{e}}}{m_{\mathrm{e}}}-\frac{B_{k \boldsymbol{Q}}^{\mathrm{h}}}{m_{\mathrm{h}}}\right)\right]^{2}, \\
f_{k}^{\prime \mathrm{e}(\mathrm{h})}=\partial f_{k}^{\mathrm{e}(\mathrm{h})} / \partial \epsilon_{k}^{\mathrm{e}(\mathrm{h})} .
\end{gathered}
$$

Resorting to the usual treatment of transforming the summation over $k$ into an integral in polar coordinates, and introducing the new variable $E=\hbar^{2} k^{2} / 2 m_{x}$, eq. (32) becomes

$$
\operatorname{Re} \varepsilon(\boldsymbol{Q})=1-\frac{2 e^{2}}{\pi \varepsilon_{0} Q^{2}}\left[2^{1 / 2}\left(\frac{m_{x}}{\hbar^{2}}\right)^{3 / 2} I_{1}(Q)+\frac{m_{x}}{\hbar^{2} Q} I_{2}(Q)\right]=0,
$$

where

$$
\begin{aligned}
& I_{1}(Q)=\int_{0}^{\infty} \mathrm{d} E \cdot E^{1 / 2}\left[f_{\mathrm{e}}^{\prime}(E)+f_{\mathrm{h}}^{\prime}(E)\right]\left[1-C^{-1}(E, Q) \operatorname{arctg} C(E, Q)\right], \\
& I_{2}(Q)=\int_{0}^{\infty} \mathrm{d} E\left[m_{\mathrm{e}} f_{\mathrm{h}}^{\prime}(E)-m_{\mathrm{h}} f_{\mathrm{e}}^{\prime}\right]\left[B_{\mathrm{e}}(E)+B_{\mathrm{h}}(E)\right] \operatorname{arctg} C(E, Q),
\end{aligned}
$$

with

$$
B_{\mathrm{e}(\mathrm{h})}(E)=A_{\mathrm{L}} \mathscr{G}\left(E+E_{\mathrm{G}}\right)+A_{\mathrm{R}}\left(E+E_{\mathrm{G}}\right) f_{\mathrm{e}(\mathrm{h})}(E),
$$




$$
\begin{gathered}
C(E, Q)=\hbar Q \sqrt{2 m_{x} E} /\left[m_{\mathrm{h}} B_{\mathrm{e}}(E)-m_{\mathrm{e}} B_{\mathrm{h}}(E)\right], \\
f_{\mathrm{e}(\mathrm{h})}^{\prime}(E)=\partial f_{\mathrm{e}(\mathrm{h})}(E) / \partial\left(\frac{m_{x}}{m_{\mathrm{e}(\mathrm{h})}} E\right),
\end{gathered}
$$

$$
f_{\mathrm{e}(\mathrm{h})}(E)=\left[1+\exp \left[\beta\left(\frac{m_{x}}{m_{\mathrm{e}(\mathrm{h})}} E-\mu_{\mathrm{e}(\mathrm{h})}\right)\right]\right]^{-1} \text {. }
$$

At room temperature $\left(T_{0}=300 \mathrm{~K}\right)$ and not too high densities of photoinjected carriers we can consider them as a nondegenerate gas consisting in the replacement in eqs. (36) of the Fermi-Dirac functions of eq. (36d) by Maxwell-Boltzmann distributions of the form

$$
f_{\mathrm{e}(\mathrm{h})}(E)=n_{\mathrm{e}(\mathrm{h})}\left[2 \pi \beta \hbar^{2} / m_{\mathrm{e}(\mathrm{h})}\right]^{3 / 2} \exp \left[-\beta \frac{m_{x}}{m_{\mathrm{e}(\mathrm{h})}} E\right],
$$

$n_{\mathrm{e}(\mathrm{h})}$ being the concentration of electrons (holes). This greatly simplifies the treatment of eq. (34), allowing for a general analytical study without resorting to specific computational calculations. In the nondegenerate regime and in the limit of small $Q$ we can write

$$
\operatorname{arctg} C(E, Q) \simeq C(E, Q),
$$

and further using that $f_{\mathrm{e}(\mathrm{h})}^{\prime}(E)=-\beta f_{\mathrm{e}(\mathrm{h})}(E)$, eq. (34) becomes

(39) $\operatorname{Re} \varepsilon(\boldsymbol{Q})=1+\frac{2 e^{2}}{\pi Q^{2} \varepsilon_{0}} \beta \int_{0}^{\infty} \mathrm{d} E D(E)\left[B_{\mathrm{e}}(E)+B_{\mathrm{h}}(E)\right] \frac{m_{\mathrm{e}} f_{\mathrm{h}}(E)-m_{\mathrm{h}} f_{\mathrm{e}}(E)}{m_{\mathrm{e}} B_{\mathrm{h}}(E)-m_{\mathrm{h}} B_{\mathrm{e}}(E)}$,

where $D(E)$ is the density of states

$$
D(E)=\frac{2 m_{x}}{\hbar^{3}}\left(2 m_{x} E\right)^{1 / 2},
$$

and the factor 2 accounts for spin degeneracy.

Taking into account that quantities $B$ (cf. eq. (36a)) are composed of two parts, one resulting from illumination, $B_{\mathrm{e}(\mathrm{h})}^{\mathrm{L}}(E)=A_{\mathrm{L}} \mathscr{G}\left(E+E_{\mathrm{G}}\right)$, and the other associated to recombination effects, $B_{\mathrm{e}(\mathrm{h})}^{\mathrm{R}}(E)=A_{\mathrm{R}}\left(E+E_{\mathrm{G}}\right) f_{\mathrm{e}(\mathrm{h})}(E)$, we consider two limiting cases that lead to simple and immediate results. First take the case when $B^{\mathrm{L}}$ gives a much smaller contribution than $B^{\mathrm{R}}$, for example illumination with a very short spectral distribution (a laser would be the extreme case of a monochromatic radiation). Then eq. (39) takes the simple form

$$
\operatorname{Re} \varepsilon(\boldsymbol{Q}) \simeq 1+\left(1 / \Lambda_{\mathrm{DH}} Q\right)^{2},
$$

where

$$
\Lambda_{\mathrm{DH}}^{-2}=4 \pi\left(n_{\mathrm{e}}+n_{\mathrm{h}}\right) e^{2} / \varepsilon_{0} k_{\mathrm{B}} T
$$

is the Debye-Hückel screening length [30], then clearly no zero of eq. (41) is possible and the homogeneous state is stable. On the other hand, if $B^{\mathrm{L}}$ predominates over $B^{\mathrm{R}}$, for example in the case of intense illumination (large $I_{0}$ ) with a large spectrum of 
frequencies and, particularly, for the cases of small values of $E_{\mathrm{G}}$ and $f_{k}$ (cf. eq. (27)), we find that eq. (39) takes the form

$$
\operatorname{Re} \varepsilon(\boldsymbol{Q})=1+\frac{8 \pi e^{2}}{\varepsilon_{0} Q^{2}} \beta \frac{m_{\mathrm{h}} n_{\mathrm{e}}-m_{\mathrm{e}} n_{\mathrm{h}}}{m_{\mathrm{h}}-m_{\mathrm{e}}} .
$$

If $n_{\mathrm{e}}=n_{\mathrm{h}}=n$, i.e. an intrinsic-type (undoped) material with only photoinjected electron-hole pairs, we recover the result of eq. (41). But, since $m_{\mathrm{h}}>m_{\mathrm{e}}$ a zero of eq. (43) can be obtained if $n_{\mathrm{h}}>n_{\mathrm{e}}$, i.e. in the case of an extrinsic-type $p$-doped material. In this case, for given $Q$ and $m_{\mathrm{e}} n_{\mathrm{h}}>m_{\mathrm{h}} n_{\mathrm{e}}$, a zero of eq. (43) follows for

$$
Q^{2}=\left[8 \pi e^{2} \beta / \varepsilon_{0}\left(m_{\mathrm{h}}-m_{\mathrm{e}}\right)\right]\left(m_{\mathrm{e}} n_{\mathrm{h}}-m_{\mathrm{h}} n_{\mathrm{e}}\right)
$$

and this implies that, writing $n_{\mathrm{h}}=n_{0}+n$ and $n_{\mathrm{e}}=n$, where $n_{0}$ is the extrinsic concentration of holes, the critical concentration $n^{*}$ of photoinjected carriers at which there follows the instability of the homogeneous state against the formation of an inhomogeneous state is given by

$$
n^{*}=\frac{m_{\mathrm{e}}-A_{0} Q^{2}}{m_{\mathrm{h}}-m_{\mathrm{e}}} n_{0},
$$

where $A_{0}=\left(m_{\mathrm{h}}-m_{\mathrm{e}}\right) \Lambda_{0}^{2}$ with $\Lambda_{0}^{2}=\varepsilon_{0} k_{\mathrm{B}} T / 8 \pi e^{2} n_{0}$, and we recall that $n$ and $I_{0}$, i.e. the concentration of photoinjected carriers and the intensity of the pumping radiation, are connected by eq. (22).

This is the idealized case when recombination effects are negligible as compared with those produced by illumination. In realistic cases both effects are present and, as we have just seen, competing one in favor of the other against the formation of the ordered pattern. Thus, eq. (39) needs be analyzed taking the full expression for functions $B$, what requires the use of numerical (computational) methods together with the specifications of the system. It ought to be noticed that our treatment implies that $n_{0}$ is high enough so as to produce a fluid of itinerant holes in the bonding band, that is to say, a concentration of carriers is required that may lead to generate screening effects enough to allow for the presence of mobile holes. This seems to be the case in proteins [31], where the concentration $n_{0}$ is of the order of $10^{18} \mathrm{~cm}^{-3}$.

Summarizing, for given $n_{0}$ there exists a critical intensity $I_{0}^{*}$ of the pumping source (which fixes $n^{*}$ ) which determines a branching point of solutions with the emergence of a steady state with spatial ordering. The latter is characterized by the set of values of $\boldsymbol{Q}$ (or wavelengths $2 \pi / Q$ ) that make contributions to the Fourier analysis of the charge density for each $I_{0}$ above $I_{0}^{*}$.

This result suggests then the possibility of a morphological transition from the homogeneous spatial distribution of carriers to a patterned structure, consisting of a superposition of steady-state charge density waves of electrons and of holes, clamped together through the effect of Coulomb interaction. It should be noticed that in the isotropic model and unbounded conditions we used in the calculations, there is no restriction on the modulus and direction of $\boldsymbol{Q}$. Since this is a longitudinal static plasma wave, it is conjecturable that $\boldsymbol{Q}$ is parallel to the electric field of the pumping electromagnetic source. Internal symmetries and boundary conditions impose limitations fixing permitted values for $Q$. 


\section{4. - Concluding remarks.}

Order and functioning of biosystems is a problem with a long history of interest attached to it, being a puzzling question in physico-chemistry. During last decades, mainly due to the work of Ilya Prigogine and collaborators, the formation and maintenance of self-organizing systems are shown to be compatible with physical laws of essentially nonlinear character $[4,5,7,32]$. These are Prigogine's dissipative structures, in which self-organization processes on a macroscopic scale follow in far-from-equilibrium conditions. The thermodynamic branch of solutions, that continually emerges from equilibrium with progressive increase of the intensity of external sources that pump energy and matter in the open system, may become-at a given threshold of excitation-unstable against the formation of a new ordered structure. This is a point of bifurcation of the solutions of the nonlinear equations of evolution that indicates a situation in which properties of one description do not imply corresponding properties of the other description. In other words, a bifurcation reflects a situation in which the incompleteness of a given mode of a system description becomes manifest requiring to be extended (as in our analysis in sect. 3) or replaced by another [33]. This question of the mode of description of natural systems is a fundamental problem of macrophysics [14].

The study presented in previous sections deals with these kinds of question. On the one hand, we have discussed the existence of a very powerful method, namely the Maxent-NSOM, that provides mechano-statistical foundations to generalized irreversible thermodynamics-a relevant discipline in the study of open biological and physico-chemical systems. This is possible whenever the system dynamics can be described (or modelled) on a microscopic level by a Hamiltonian approach. Then one can construct a nonlinear transport theory that provides the equations of evolution for the basic set of variables that are used to describe the macroscopic state of the system. These nonlinear transport equations are the generalization of the phenomenological kinetic laws of generalized irreversible thermodynamics, in the sense that, in principle, they contain all orders of nonlinearity and give explicit expressions at the microscopic level for the transport coefficients, in terms of the actual nonequilibrium state of the system. Points of bifurcation of solutions (and formation of dissipative structures) can then be determined and any state along any branch of solutions can be characterized and studied in detail.

We applied the method to study the possibility that a quite simplified model of an open biosystem may show a transition to a dissipative structure, in the form of a spatially ordered macrostate, when under the action of external illumination. We have shown that in the case of a $p$-doped sample, as it seems to be the case with certain proteins [31], a bifurcation point follows at a threshold of intensity of the source of radiation, when the homogeneous electronic steady state becomes unstable against the formation of a stationary charge density wave (SCDW). In the treatment of the problem we performed we kept fixed the positive background of ionic charges. It is quite plausible that the attractive interaction of the latter with the electrons will tend to allow the ions to follow the electronic SCDW, thus producing an ordered pattern in the whole system (a conformational-like transition).

As discussed in sect. 3 there exists a competition between illumination and recombination effects and, as a result, the transition from the homogeneous to the patterned distribution follows after the control parameter, consisting of the intensity of the source of radiation, has reached a critical value. The homogeneous distribution 
is no longer stable and a SCDW is formed, which develops an increasing amplitude with increasing pump intensity. It ought to be noticed that formation of the SCDW depends on the external influence, but the pattern itself is determined by the internal characteristics of the system "codified" in its Hamiltonian. Therefore, reproducible pattern formation is guaranteed, although the pattern may have been initiated in different ways.

Summarizing, we have shown-through the use of a quite simple model-how biological pattern formation may follow as a result of nonlinear kinetic effects that describe production and decay rates involving autocatalytic effects. We are in the presence of an example of the emerging theory of complexity, when nonlinear terms involving strong positive feedback processes lead to unexpected, and, in a sense, counterintuitive phenomena. Finally, we would like to once again emphasize the usefulness of the very powerful theoretical tool that is the concise and practical NSOM for providing a rigorous and suitable framework, based on first principles, for dealing with dissipative many-body systems in far-from-equilibrium conditions. It provides at the microscopic mechano-statistical level means for the rigorous characterization and study of dissipative structures in complex systems. In the present paper we have emphasized the possible influence of electrons in such type of biological phenomena. In this context our results seem to be related to Szent-Györgyi's view that hole mobility in the extended ground states of protein molecules may lead to the building of higher structures and to control all differentiation, namely that electronic properties may be responsible for the activity and subtlety of many biological functions [34]. As a final word we stress that we have explicitly used photoexcitation of electrons from the bonding to the antibonding energy levels, but the final result is depending only on the existence of the resulting population inversion, and therefore, the phenomenon may also be expected for any other type of excitation process capable of producing such population inversion.

The authors are Brazilian National Research Council (CNPq) research fellows. We also thank Dr. T. Tomé (USP) for her collaboration in the early stages of this work.

\section{REFERENCES}

[1] Meinhardt H., Rep. Prog. Phys., 55 (1992) 797.

[2] Cross M. C. and Hohenberg P. C., Rev. Mod. Phys., 65 (Part II) (1993) 851.

[3] Prigogine I., Nature, 246 (1973) 67; also Int. J. Quantum Chem. Symp., 9 (1975) 443.

[4] GlansdorfF P. and Prigogine I., Thermodynamics of Structure, Stability, and Fluctuations (Interscience-Wiley, New York) 1971.

[5] Nicolis G. and PRIGOGINE I., Self-Organization in Nonequilibrium Systems (Interscience-Wiley, New York) 1977.

[6] Normand C., Pomeau Y. and Velarde M. G., Rev. Mod. Phys., 49 (1977) 581; also KREUzER H. J., Nonequilibrium Thermodynamics and its Statistical Foundations (Clarendon, Oxford) 1981.

[7] Prigogine I., Nicolis G. and Babloyantrz A., Phys. Today, 25 (1972) 23 and 38. 
[8] HAKEN H., Synergetics (Springer, Berlin-Heidelberg) 1978.

[9] Turing A. M., Philos. Trans. R. Soc. London, Ser. B, 237 (1952) 37.

[10] Robertson B., Phys. Rev., 144 (1966) 151; in The Maximum Entropy Formalism, edited by R. D. Levine and M. TRibus (MIT Press, Cambridge, Mass., 1978).

[11] Zubarev D. N., Neravnovesnaia Statisticheskaia Termodinamika (Izd. Nauka, Moscow 1971), English transl., Nonequilibrium Statistical Thermodynamics (Consultants Bureau, New York) 1974, Chapt. IV.

[12] Luzzi R. and Vasconcellos A. R., Fortschr. Phys./Prog. Phys., 38 (1990) 887.

[13] E.g., Casas VazQuez J., Jou D. and Lebon G. (Editors), Recent Developments in Nonequilibrium Thermodynamics (Springer, Berlin-Heidelberg) 1984; JoU D., CASAS VazQuez J. and Lebon G., Rep. Prog. Phys., 51 (1988) 1105.

[14] Vasconcellos A. R., Luzzi R. and Garcia-Colin L., Phys. Rev. A, 43 (1991) 6622, 6633.

[15] Ramos J. G., Vasconcellos A. R. and Luzzi R., Phys. Rep. (submitted).

[16] JaYnes E. T., in Frontiers of Nonequilibrium Statistical Physics, edited by G. T. Moore and M. O. SCULly (Plenum, New York) 1983; also in E. T. Jaynes' Papers on Probability, Statistics and Statistical Physics, edited by D. Rosenkrantz (Reidel, Dordrecht) 1983.

[17] Anderson P. W., Phys. Today, 45 (1992) 9.

[18] E.g., Garcia Colin L. S., Vasconcellos A. R. and Luzzi R., J. Non-Equilib. Thermodynamics, 19 (1993) 24: J. Kestin's Memorial Issue.

[19] Lauck L., Vasconcellos A. R. and Luzzi R., Physica A, 168 (1990) 789.

[20] Bogoliubov N. N., in Studies in Statistical Mechanics I, edited by J. DE BoER and G. E. UHLENBECK (North-Holland, Amsterdam) 1962. For additional discussions and applications see: UhlENBECK G. E., in Lectures in Statistical Mechanics, edited by M. KAC (Am. Math. Soc., Providence, R.I.) 1963; Buishvili L. L. and Zviadadze M. D., Physica, 59 (1972) 697; Vasconcellos A. R., Algarte A. C. and Luzzi R., Physica A, 166 (1990) 517.

[21] MonI H., Prog. Theor. Phys., 33 (1965) 423.

[22] Peletminskit S. V. and Yatsenko A. A., Ž. Éksp. Teor. Fiz., 53 (1967) 1327 (Sov. Phys. JETP, 26 (1968) 773).

[23] Luzzi R. and Vasconcellos A. R., J. Stat. Phys., 23 (1980) 539.

[24] Kalashnikov V. P., Teor. Mat. Fiz., 34 (1978) 412 (Theor. Math. Phys. (USSR), 34 (1978) 263).

[25] Tomé T., Vasconcellos A. R. and Luzzi R., Physica B, 144 (1987) 376.

[26] Pines D., The Many-Body Problem (Benjamin, Reading, Mass.) 1961; Ph. Nozières and D. PINES, Quantum Fluids (Benjamin, Reading, Mass.) 1966.

[27] E.g., BlaCKemore L. S., Semiconductor Statistics (Pergamon, Oxford) 1962.

[28] Davies H. T., Introduction to Nonlinear Differential and Integral Equations (Dover, New York) undated.

[29] E.g., LANDAU L. D. and LifShiTZ I. E., Electrodynamics of Continuous Media (Addison-Wesley, Reading, Mass.) 1960.

[30] E.g., Ziman J. M., Electrons and Phonons (Clarendon Press, Oxford) 1960.

[31] Pethig R., Int. J. Quantum Chem. Symp., 5 (1978) 159.

[32] Prigogine I., From Being to Becoming (Freeman, San Francisco) 1980.

[33] Rosen R., in Bifurcation Theory and Applications, edited by O. GUREL and O. E. RössLER (New York Academy of Sciences, New York) 1979.

[34] Szent-GyörgYi A., Nature, 148 (1941) 157; Science, 93 (1941) 609; SzEnT-GyöRGYi A. and McLaughlin J. A., Int. J. Quantum Chem. Symp., 5 (1978) 137. 